\title{
Non-Perturbative Treatment of Quantum Mathieu Oscillator
}

\author{
Mohammed Janati Idrissi, Abdelaziz Fedoul, Salaheddine Sayouri
}

Laboratory of Theoretical and Applied Physics, Faculty of Sciences Dhar Al Mahraz, University of Sidi Mohamed Ben Abdellah, Fez, Morocco

Email: afedoul@yahoo.fr, ssayouri@gmail.com,jan123med@gmail.com

How to cite this paper: Idrissi, M.J., Fedoul, A. and Sayouri, S. (2020) Non-Perturbative Treatment of Quantum Mathieu Oscillator. Journal of Applied Mathematics and Physics, 8, 698-709.

https://doi.org/10.4236/jamp.2020.84054

Received: March 4, 2020

Accepted: April 12, 2020

Published: April 15, 2020

Copyright (C) 2020 by author(s) and Scientific Research Publishing Inc. This work is licensed under the Creative Commons Attribution International License (CC BY 4.0)

http://creativecommons.org/licenses/by/4.0/

\begin{abstract}
We study the evolution in time of the quantum Mathieu oscillator (QMO), according to the motion of a charged particle in a radio frequency Paul trap. We adopt non-perturbative treatment based on the quantized Floquet formalism together with the resonating averages method (RAM). We prove that we can develop solutions of the time-dependent Schrödinger equation of such a system, in terms of the simple harmonic oscillator wave functions. Numerical simulations of the analytical results are performed to show the coherence and the squeezed proprieties of the wave-packet of this system.
\end{abstract}

\section{Keywords}

Floquet Theorem, Resonating Averages, Mathieu Oscillator, Paul Trap

\section{Introduction}

Numerous mathematical and physical studies have been devoted to the construction of robust framework for solving the time-dependent Schrödinger equation of quantum systems [1] [2]. In this regard, several approaches were developed, among them there are the usual perturbation theory [3], Lie algebraic method, generalized invariant operator and path integral methods [3] [4] [5] [6], which have been applied to some special cases of time-dependent quantum systems, to find the time evolution operator, which in turn enables to write the explicit expressions of the wave functions and the correspondent energies of these systems. In particular, in the regime of strong laser-matter interaction problems, a non-perturbative approach, based on the quantization version of the Floquet theorem has been established and applied to schemes of physical systems with periodic Hamiltonians [7] [8] [9]. Notably, this was used to give explanation of multiphoton processes in intense laser fields [10], selective excitation of molecu- 
lar vibrational states using short laser pulses [11]. It is worth noticing that mathematical contributions of the above-mentioned researches and physical challenge are dealing with the interpretation of experimental results and the description of the nature of various physical phenomena such as quantum optic, quantum chemistry, cosmology [1] [4] [6], etc.

Our motivation consists to the application of this non-perturbative approach, especially to a system driven by a periodic time-varying frequency. Such a system has already been studied by Lemos to give a theoretical approach of the expansion of the universe [12], and by Paul to describe the motion of a charged particle in an oscillating electric field [13] [14] [15]. Floquet theory is an efficiency tool for strong time-periodic interaction, and consequently for which the usual stationary states, solutions to the time-dependent Schrödinger equation when no external field acts, disappear. The adiabatic invariance principle formula established by Breuer and Holthaus [16], is then used to give a physical interpretation to the derived quantum states. These quantum steady states or Floquet states constitute the most probable states, and provide a useful dynamical description induced by the time-periodic interaction.

In previous works, we have applied our non-perturbative method, to the cases of the forced harmonic oscillator, and to some driven anharmonic potentials models [17] [18]. Indeed, the RAM [19] provides a useful tool for constructing the evolution operators in a whole resonance zone, which enabled us to obtain readily the wave functions and the associated quasi-energies of these systems. Therefore, some physical properties of these results were explored and exploited. The aim of the present paper is to apply this approach, to first and second ameliorated orders approximation, to the quantum Mathieu oscillator (QMO) model. We have determined the time-evolution operators, the Floquet states, the wave functions and the corresponding energies, and we have shown that the Floquet states of the same parity are coupled in the presence of the interaction Hamiltonian. Moreover, we have verified the Heisenberg uncertainty principle for all time and we have performed numerical evaluations, for the operational parameters that appeared in the analytical expressions.

The paper is structured as follows. In Section 2, we give a review of the theoretical strategy of our method. Section 3, is focused its application to the QMO, and gives the established analytics results, as well as the numerical simulations for the parameters values. In Section 4, we give the conclusions and outlook.

\section{Theoretical Strategy [17] [18]}

The Schrödinger equation of a quantum system with a time-dependent Hamiltonian $H(t)$ can be written in terms of the time-evolution operator $U(t, 0)$ such as,

$$
\begin{gathered}
\mathrm{i} \hbar \frac{\mathrm{d} U(t, 0)}{\mathrm{d} t}=H(t) U(t, 0) \\
H(t)=H_{0}+\lambda H^{\prime}(t)
\end{gathered}
$$


where $H_{0}$ is the Hamiltonian of the unperturbed system, and $H^{\prime}(t)$ the interaction Hamiltonian, which amplitude $\lambda$ is taken as being very small, and $U(t, 0)$ is such that

$$
|\psi(t)\rangle=U(t, 0)|\psi(0)\rangle
$$

where $|\psi(t)\rangle$ describes the system state at the time $t$, and $|\psi(0)\rangle$ is an arbitrary initial state.

In the case of a time-periodic interaction, the Floquet theorem proves the existence of a couple of operators $(R, T(t))$, where $R$ is a constant Hermitian operator, and $T(t)$ is a periodic unitary operator of the same period as $H^{\prime}(t)$ so that the formulation of the time-evolution operator $U(t)$, solution of Equation (1), is given in the form [7] [8] [9]

$$
U(t)=T(t) \mathrm{e}^{\frac{-\mathrm{i} R t}{\hbar}}
$$

and the initial condition $U(0)=1$.

Replacing the unitary transformation $T(t)$ on Equation (1) gives rise to the usual time-independent Schrödinger equation of the operator $R$ (so-called the reduced representation of the system), such as

$$
\begin{gathered}
\mathrm{i} \hbar \frac{\mathrm{d}\left|\phi_{n}(t)\right\rangle}{\mathrm{d} t}=R\left|\phi_{n}(t)\right\rangle \\
\left|\phi_{n}(t)\right\rangle=\mathrm{e}^{\frac{-\mathrm{i} E_{n} t}{\hbar}}|n\rangle
\end{gathered}
$$

where $\left|\phi_{n}(t)\right\rangle$ are the eigenvectors of the operator $R$, corresponding to the eigenvalues $E_{n}$, and where $|n\rangle$ are the eigenstates of the Hamiltonian $H_{0}$.

The resolution of Equation (5) leads to obtain the Floquet states, which are canonical solutions of the time-dependent Schrödinger equation of the system [8]; they are defined as being

$$
\left|\psi_{n}(t)\right\rangle=T(t)\left|\phi_{n}(t)\right\rangle
$$

These derive quantum states constitute a complete set of bound states of the system, and do not depend on the choice of the couple $(R, T(t))$. One shows otherwise that these are the invariant adiabatic states. Then, the global state $|\psi(t)\rangle$ describing the quantum system can be expanded in terms of a linear combination with time-independent coefficients of the $\left|\psi_{n}(t)\right\rangle$.

But, no general method is valid to calculate the operators $R$ and $T(t)$. To determine these two operators, we used the technique based on the resonating averages method (RAM) elaborated by Lochak [19]. This method is applied in the interaction picture of $H^{\prime}(t)$ which is written with the summation of the averaging term $\bar{H}_{I}(t)$ and the oscillating term $\frac{\mathrm{d} \tilde{H}_{I}(t)}{\mathrm{d} t}$. It permits to find an approximate solution of Equation (1), to a certain order in power of $\lambda$. This method may be compared to the customary rotating wave approximation [20].

Determination of first and second orders ameliorated solutions and a com- 
parison with the formulation of Equation (4), enable us to obtain the first and second orders of Floquet operators couples, thence we can use Equation (5) and Equation (6) to set up Floquet states, and the correspondents quasi-energies respectively.

\section{Application to the Quantum Mathieu Oscillator}

\subsection{Analytical Results}

The differential equation of motion without damping of the spatial coordinates (in one dimension), for a charged particle submitted to a time varying electric field is given in classical mechanics in the general form [13] [15] [21]

$$
\ddot{q}+\Omega^{2}(t) q=0
$$

By choosing the potential function (or time varying frequency $\Omega(t)$ ) such that,

$$
\Omega^{2}(t)=\omega_{0}^{2}(\delta+\lambda \cos (2 \omega t))
$$

where $\omega_{0}$ is the unperturbed oscillator frequency, $\omega$ is the driven oscillation frequency, and where $\lambda$ is the perturbation amplitude taken as been very small.

This model supposes that a charged particle is governed by a Mathieu equation also called Mathieu oscillator, is a particularly important case, which enables to describe the motion of a charged particle in a linear radio frequency Paul trap device [13].

Without loss of generality of the model, we adopt the parameter of the trap $\delta=1$.

The Hamiltonian of such oscillator according to Equation (7) and Equation (8) is given as follows,

$$
H(t)=\frac{p^{2}}{2 m}+\frac{1}{2} m \Omega^{2}(t) q^{2}
$$

By using the $\mathrm{SU}(1,1)$ generators group elements $J_{1}$ and $J_{3}$, which are expressed in terms of the conjugate variables $q$ and $p$ and in terms of the creation and the annihilation operators according to

$$
\begin{aligned}
& J_{1}=\frac{\left(m \omega_{0}\right)^{2} q^{2}-p^{2}}{4 m \hbar \omega_{0}}=\frac{1}{4}\left(a^{2}+a^{+2}\right) \\
& J_{3}=\frac{\left(m \omega_{0}\right)^{2} q^{2}+p^{2}}{4 m \hbar \omega_{0}}=\frac{1}{2}\left(a^{+} a+\frac{1}{2}\right)
\end{aligned}
$$

Leads to write the Hamiltonian $H(t)$ of this system under the form [22]

$$
H(t)=2 \hbar \omega_{0} J_{3}+\lambda \hbar \omega_{0} \cos (2 \omega t)\left[J_{1}+J_{3}\right]
$$

where $q$ and $p$ satisfy the commutation relation $[q, p]=\mathrm{i} \hbar$.

Using Equation (10) and Equation (11), yields to write $H^{\prime}(t)$ in Equation (2) as

$$
H^{\prime}(t)=\frac{\hbar \omega_{0}}{4} \cos (2 \omega t)\left(a^{+2}+a^{2}+2 a^{+} a+1\right)
$$

Note that the expression of $H^{\prime}(t)$ is similar to that published by Profilo et al. 
[5] by doing the first order development of $\Omega(t)$ given in Eqution (8), and using the invariant operator method in the framework of the Lie Group.

The RAM applied to the interaction picture form [17] [18] of $H^{\prime}(t)$ gives

$$
\begin{gathered}
\bar{H}_{I}(t)=0 \\
\tilde{H}_{I}(t)=\eta_{0}(t)\left(2 a^{+} a+1\right)+\eta_{1}^{\star}(t) \mathrm{e}^{2 i \omega_{0} t} a^{+2}+\eta_{1}(t) \mathrm{e}^{-2 i \omega_{0} t} a^{2}
\end{gathered}
$$

where

$$
\begin{gathered}
\eta_{0}(t)=\frac{\hbar \omega_{0} \sin (2 \omega t)}{8 \omega} \\
\eta_{1}(t)=\frac{\hbar \omega_{0}}{8} \frac{\omega \sin (2 \omega t)-i \omega_{0} \cos (2 \omega t)}{\omega^{2}-\omega_{0}^{2}}
\end{gathered}
$$

and where $\eta_{1}^{\star}(t)$ is the complex conjugate of $\eta_{1}(t)$.

Using the expressions of the evolution operator given by the (RAM) [17] [18], enabled us to obtain the first and second ameliorated solutions terms of quasi-energies, Floquet states and wave-functions as

$$
\begin{gathered}
{ }^{(1 a)} E_{n}=\hbar \omega_{0}\left(n+\frac{1}{2}\right) \\
\left.\left.\right|^{(1 a)} \psi_{n}(t)\right\rangle=\mathrm{e}^{\frac{-\mathrm{i}^{(1 a)} E_{n} t}{\hbar}}\left[|n\rangle-\frac{\mathrm{i} \lambda}{\hbar}\left[(2 n+1) \eta_{0}(t)|n\rangle\right.\right. \\
\left.\left.+\sqrt{n(n-1)} \eta_{1}(t)|n-2\rangle+\sqrt{(n+1)(n+2)} \eta_{1}^{\star}(t)|n+2\rangle\right]\right] \\
{ }^{(2 a)} E_{n}=\hbar \omega_{0}\left(n+\frac{1}{2}\right)\left[1+\frac{\lambda^{2} \omega_{0}^{2}}{16\left(\omega^{2}-\omega_{0}^{2}\right)}\right] \\
\left|\psi_{n}(t)\right\rangle= \\
\quad \mathrm{e}^{\frac{-\mathrm{i}^{(2 a)} E_{n} t}{\hbar}}\left[k_{-4}|n-4\rangle+k_{-2}|n-2\rangle+k_{0}|n\rangle\right. \\
\left.+k_{+2}|n+2\rangle+k_{+4}|n+4\rangle\right] \\
{ }^{(2 a)} \psi_{n}(q, t)=\mathrm{e}^{\frac{-\mathrm{i}^{(2 a)} E_{n} t}{\hbar}}\left[k_{-4} \varphi_{n-4}(q)+k_{-2} \varphi_{n-2}(q)+k_{0} \varphi_{n}(q)\right. \\
\left.+k_{+2} \varphi_{n+2}(q)+k_{+4} \varphi_{n+4}(q)\right]
\end{gathered}
$$

The different coefficients $k_{ \pm i}$ are given by

$$
\begin{gathered}
k_{-4}=\frac{-\lambda^{2}}{2 \hbar^{2}} \eta_{1}^{2}(t) \sqrt{n(n-1)(n-2)(n-3)} \\
k_{-2}=\left[-\frac{\mathrm{i} \lambda}{\hbar} \eta_{1}(t)+\frac{\lambda^{2}}{2 \hbar^{2}}\left(\eta_{2}(t)-2(2 n-1) \eta_{0}(t) \eta_{1}(t)\right)\right] \sqrt{n(n-1)} \\
k_{0}=1-\frac{\mathrm{i} \lambda}{\hbar}(2 n+1) \eta_{0}(t) \\
+\frac{\lambda^{2}}{2 \hbar^{2}}\left[(2 n+1) \eta_{3}(t)-(2 n+1)^{2}\left(\eta_{0}^{2}(t)+\frac{1}{2}\left|\eta_{1}\right|^{2}\right)-\frac{3}{2}\left|\eta_{1}\right|^{2}\right]
\end{gathered}
$$




$$
\begin{gathered}
k_{+2}=\left[-\frac{\mathrm{i} \lambda}{\hbar} \eta_{1}^{\star}(t)-\frac{\lambda^{2}}{2 \hbar^{2}}\left(\eta_{2}^{\star}(t)+2(2 n+3) \eta_{0}(t) \eta_{1}^{\star}(t)\right)\right] \sqrt{(n+1)(n+2)} \\
k_{+4}=\frac{-\lambda^{2}}{2 \hbar^{2}} \eta_{1}^{\star 2}(t) \sqrt{(n+1)(n+2)(n+3)(n+4)}
\end{gathered}
$$

where

$$
\begin{gathered}
\eta_{2}(t)=\frac{-\left(\hbar \omega_{0}\right)^{2}\left[3 \omega_{0}^{2} \omega \cos (4 \omega t)-\omega\left(4 \omega^{2}-\omega_{0}^{2}\right)+\mathrm{i} \omega_{0}\left(\omega_{0}^{2}+2 \omega^{2}\right) \sin (4 \omega t)\right]}{32 \omega\left(4 \omega^{2}-\omega_{0}^{2}\right)\left(\omega^{2}-\omega_{0}^{2}\right)} \\
\eta_{3}(t)=\frac{-\mathrm{i} \hbar^{2} \omega_{0}^{3}}{64 \omega\left(\omega^{2}-\omega_{0}^{2}\right)} \sin (4 \omega t) \\
\varphi_{n}(q)=\left(\frac{\alpha}{\pi}\right)^{\frac{1}{4}} \frac{\mathrm{e}^{\frac{-\alpha q^{2}}{2}}}{\sqrt{2^{n} n !}} H_{n}(\sqrt{\alpha} q)
\end{gathered}
$$

$\varphi_{n}(q)$ is the wave function of the simple oscillator, $H_{n}(\sqrt{\alpha} q)$ are the Hermite polynomials and the parameter $\alpha=\frac{m \omega_{0}}{\hbar}$.

We note that the correction effects on quasi-energies appear only from the second order term of the approximation and depend on the quantum number $\mathrm{n}$ and the parameters ( $\lambda$ and $\omega$ ) of the variable frequency $\Omega(t)$. Therefore, the Floquet states expression shows that the transitions between these Floquet states $\left(\left|\psi_{n}(t)\right\rangle\right.$ and $\left.\left|\psi_{k}(t)\right\rangle\right)$ are suppressed when the levels do not have the same parity (i.e. $n=k+1)$.

The difference between two adjacent second order Floquet levels is given by,

$$
\Delta E={ }^{(2 a)} E_{n+1}-{ }^{(2 a)} E_{n}=\hbar \omega_{0}\left(1+\frac{\lambda^{2} \omega_{0}^{2}}{16\left(\omega^{2}-\omega_{0}^{2}\right)}\right)
$$

The Equation (23) shows that all levels are shifted equally; therefore the energy levels of the system remain equidistant.

By using some identities and Equation (18), we can easily compute the expectation values of $q, p, q^{2}$ and $p^{2}$ for any level. Neglecting the coefficients of $\left(\lambda^{3}\right.$ and $\lambda^{4}$ ), permits to write the corresponding fluctuations in $q$ and $p$ as follows,

$$
\begin{gathered}
{ }^{(2 a)} \Delta q=\sqrt{\frac{(2 n+1) \hbar}{2 m \omega_{0}}}\left[1-4 \lambda \operatorname{Im} \eta_{1}(t)+\lambda^{2}\left(8\left|\eta_{1}\right|^{2}-2\left[\operatorname{Re}_{2}(t)+4 \eta_{0}(t) \operatorname{Re} \eta_{1}(t)\right]\right)\right]^{\frac{1}{2}} \\
{ }^{(2 a)} \Delta p=\sqrt{\frac{(2 n+1) \hbar m \omega_{0}}{2}}\left[1+4 \lambda \operatorname{Im} \eta_{1}(t)+\lambda^{2}\left(8\left|\eta_{1}\right|^{2}+2\left[\operatorname{Re}_{2}(t)+4 \eta_{0}(t) \operatorname{Re} \eta_{1}(t)\right]\right)\right]^{\frac{1}{2}}
\end{gathered}
$$

Then one deduce the second order uncertainty relation such as

$$
{ }^{(2 a)}(\Delta q \Delta p)_{n, n}=(2 n+1) \frac{\hbar}{2}\left[1+\frac{\lambda^{2} \omega^{2} \omega_{0}^{2}}{8\left(\omega^{2}-\omega_{0}^{2}\right)^{2}} \sin ^{2}(2 \omega t)\right]
$$

It is easily seen that the fluctuation $\Delta q$ and $\Delta p$ product shows that for all 
times $t$ and for any level, the Heisenberg uncertainty principle is satisfied, as well as presents an oscillating correction term due to the form of $\Omega(t)$.

\subsection{Numerical Results}

As an illustration we carried out numerical simulations of the established analytical expressions, for different values of the parameters. From Equation (19), we can compute the expressions of the probability density associated to the wave-packet of the system. As an example, for the values of the parameters taken to be: $\lambda=0.01,2 \Pi \hbar=m=1$ and $\omega_{0}=8 \omega$.

We give on Figure 1 and Figure 2, the plots of the wave-packet, in the case where the number state are $n=0$ and $n=7$.

On Figures 3-5, we give the simulation plots of $\left(q_{0}^{(2 a)} \Delta q\right),\left(p_{0}^{(2 a)} \Delta p\right)$ and $\frac{2}{\hbar}{ }^{(2 a)}(\Delta q \Delta p)_{7,7}$ as functions of $t$, for diferent values of $\frac{\omega_{0}}{\omega}$, $\left(\frac{\omega_{0}}{\omega}=1.1 ; 1.15 ; 1.2 ; 1.4\right)$, where the constants factor $q_{0}$ and $p_{0}$ are given by $q_{0}=\sqrt{\frac{2 m \omega_{0}}{\hbar}}$ and $p_{0}=\sqrt{\frac{2}{\hbar m \omega_{0}}}$.

Figure 1 and Figure 2 show that, when departing from an initial coherent state, the wave-packets $\left(\left|{ }^{(2 a)} \psi_{0}(q, t)\right|^{2}\right)$ and $\left(\left|{ }^{(2 a)} \psi_{7}(q, t)\right|^{2}\right)$ propagate with small displacement of the maximums for different instants, keeping its form and that the curve presents the regular nodal character. Therefore, the dynamic localization of the wave-packet is verified.

Figure 3 and Figure 4 show that there is a decrease of $\Delta q$ at the expense of an increase of $\Delta p$, as functions of time and for different values of $\frac{\omega_{0}}{\omega}$ and indicate that the maximum of these fluctuations varies as a function of $\frac{\omega_{0}}{\omega}$. Thus the squeezing property of Floquet states $\left|\psi_{n}(t)\right\rangle$ is apparent here. Figure 5 also indicates that the uncertainty product is periodic in time and increases strongly to reach its maximums when $\frac{\omega_{0}}{\omega} \approx 1$. Thence, the trapped charged particles

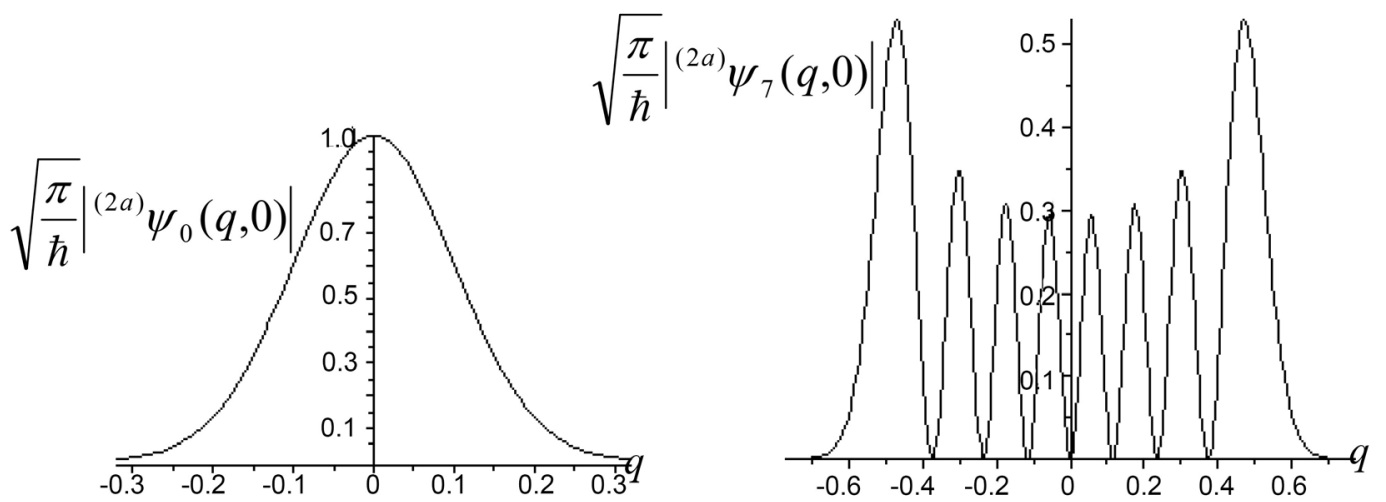

Figure 1. The probability density vs. $q$ and $t=0$, for $n=0$ and $n=7$. 


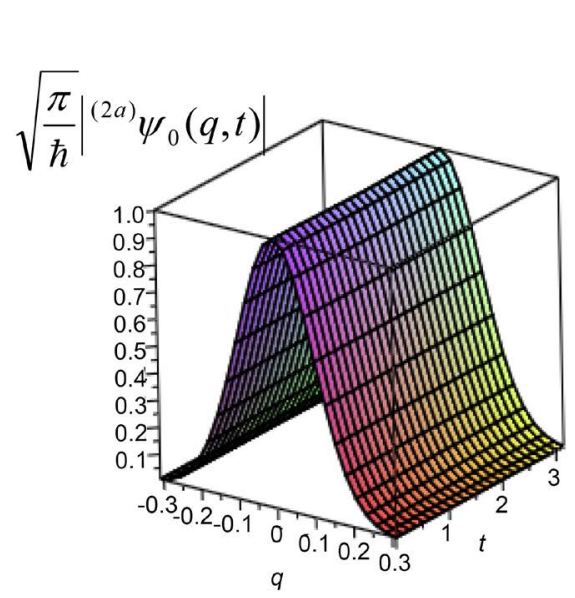

$$
\sqrt{\frac{\pi}{\hbar}}\left|(2 a) \psi_{7}(q, t)\right|
$$

Figure 2. The evolution of the probability density vs. $q$ and $t$, for $n=0$ and $n=7$.
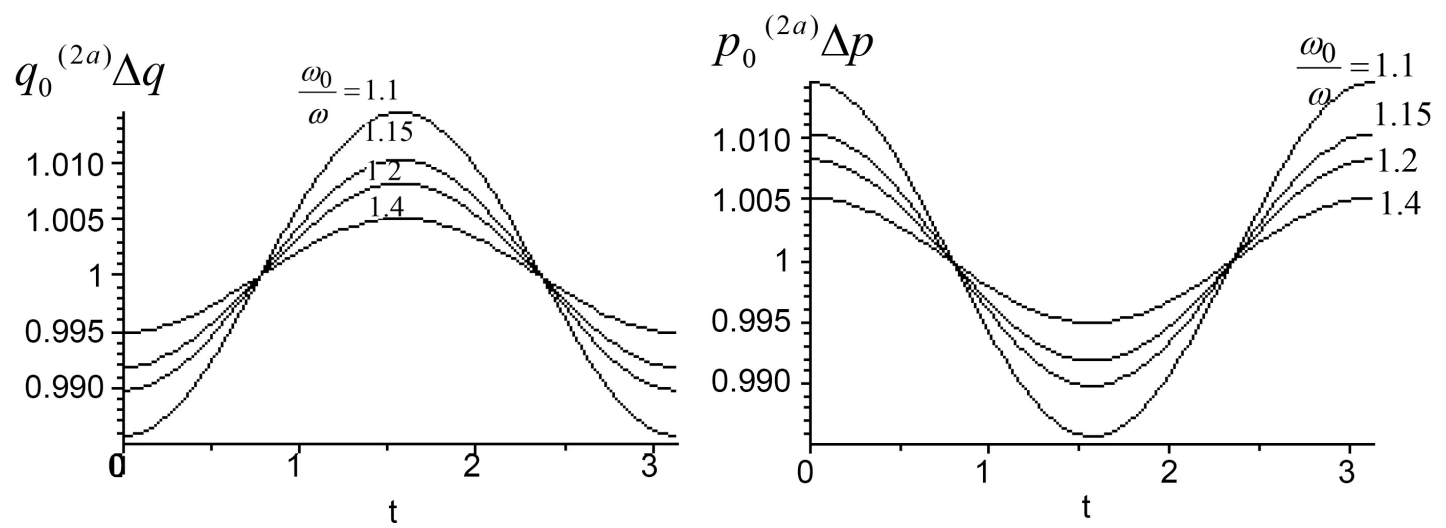

Figure 3. The variation of $q_{0}^{(2 a)} \Delta q$ and $p_{0}^{(2 a)} \Delta p$ vs. $t$ for $n=0$ and for different values of $\frac{\omega_{0}}{\omega}$.
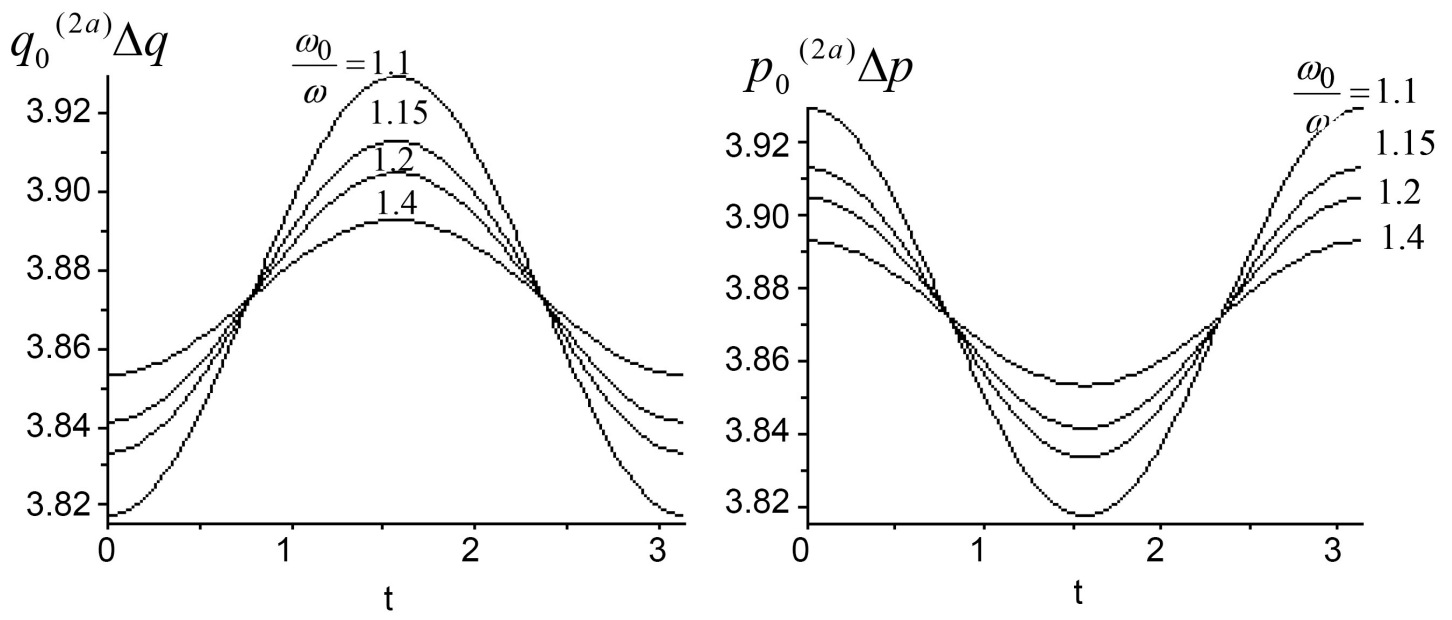

Figure 4. The variation of $q_{0}^{(2 a)} \Delta q$ and $p_{0}^{(2 a)} \Delta p$ vs. $t$ for $n=7$ and for different values of $\frac{\omega_{0}}{\omega}$.

oscillate harmonically along each spatial axis at the secular frequency $\omega$. Our results are agreed with those published by other authors [3] [15] [23] [24]. 


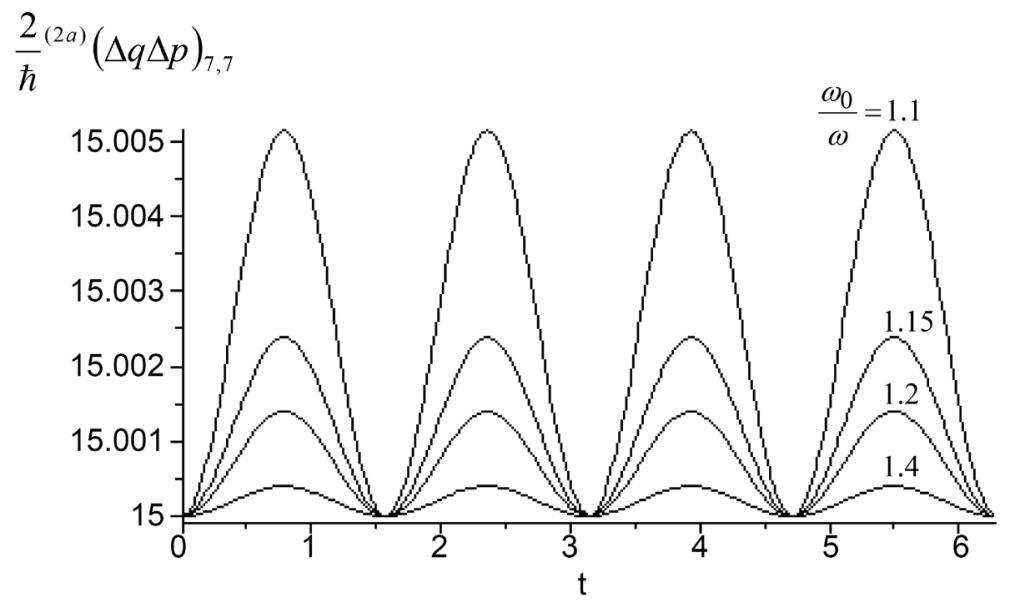

Figure 5. The variation of $\frac{2}{\hbar}^{(2 a)}(\Delta q \Delta p)_{7,7}$ vs. $t$ for different values of $\frac{\omega_{0}}{\omega}$.

\section{Conclusions and Outlook}

Floquet formalism and the RAM have been successfully used to solve the quantum Mathieu oscillator problem. Floquet states and the corresponding eigenstates and eigenvalues have been expressed to first and second orders approximation. We have shown that the transitions are valid only between Floquet states with the same parity; this is due to the form of the interaction Hamiltonian $H^{\prime}(t)$ of the system. This approach seems for us easier in constructing the quantum states of the studied system in comparison with others [3] [4] [5]. Moreover, we have expressed the uncertainty principle and verified its validity for all time. Numerical computations have also been performed to illustrate the dynamic localization of the wave packet of the system.

Let us argue that our analysis indicates that the effect of the periodic variation of the interaction is to generate the coherence and the squeezed proprieties of the wave-packet of this system and that the effect of the periodic variation of $\Omega(t)$ involves an oscillating correction of the uncertainty relation. In addition a divergent character of this correction at a primary resonance frequency $\omega=\omega_{0}$ of the system is observed.

Let us note that in the absence of the perturbation, we recover the uncertainty relation of the simple oscillator. Let us also note that if we consider the parameter $\delta=0$, we recover the results established for the forced harmonic oscillator [17], signifying that the trapped charged particles can be seen as harmonic oscillator with a time modulated frequency $\Omega(t)$. Our studies argued that the Paul trapped ions may constitute a good promising candidate for physical realization of a quantum computer.

Comparisons of our results, with works published by other authors who have used different methods [3] [15] [23] [24] reveal a good concordance. Our results may play an interesting complementary role in the treatment of various harmonically confined system. We project to apply our approach to a nonlinear driven Duffing oscillator. 


\section{Conflicts of Interest}

The authors declare no conflicts of interest regarding the publication of this paper.

\section{References}

[1] Klauder, R.J. and Skagerstam, B.S. (1985) Coherent States-Application in Physic and Mathematical Physics. World Scientific, Singapore. https://doi.org/10.1142/0096

[2] Cohen-Tannoudji, C., Diu, B. and Laloë, F. (1973) Mécanique Quantique T2. Hermann, Paris, 1083-1224.

[3] Lo, C.L. (1990) Squeezing by Tuning the Oscillator Frequency. Journal of Physics A: Mathematical and Theoretical, 23, 1155-1165. https://doi.org/10.1088/0305-4470/23/7/021

[4] Lewis Jr., H.R. and Reinsenfeld, W.B. (1969) An Exact Quantum Theory of the Time Dependent Harmonic Oscillator and of a Charged Particle Time Dependent Electromagnetic Field. Journal of Mathematical Physics, 10, 1458-1473. https://doi.org/10.1063/1.1664991

[5] Profilo, G. and Soliana, G. (1991) Group-Theoretical Approach to the Classical and Quantum Oscillator with Time-Dependent Mass and Frequency. Physical Review, 44, 2057-2065. https://doi.org/10.1103/PhysRevA.44.2057

[6] Yeon, K.H., Kim, H.J., Um, C.I., George, T.F. and Pandey, L.N. (1994) Wave Function in the Invariant Representation and Squeezed-State Function of the Time-Dependent Harmonic Oscillator. Physical Review, 50, 1035-1039. https://doi.org/10.1103/PhysRevA.50.1035

[7] Floquet, G. (1883) Sur les éqautions différentielles linéaires à coefficients périodiques. Annales scientifiques de P École normale supérieure, 12, 47-88. https://doi.org/10.24033/asens.220

[8] Shirley, J.H. (1965) Solution of the Schrödinger Equation with a Hamiltonian Periodic in Time. Physical Review, 138, 979-987. https://doi.org/10.1103/PhysRev.138.B979

[9] Chu, S.I. and Telnov, D.A. (2004) Beyond the Floquet Theorem: Generalized Floquet Formalisms and Quasienergy Methods for Atomic and Molecular Multiphoton Processes in Intense Laser Fields. Physics Reports, 390, 1-131. https://doi.org/10.1016/j.physrep.2003.10.001

[10] Huang, Y. and Chu, S.I. (1994) A Stationary Treatment of Time-Dependent Hamiltonian by the Many-Mode Floquet Formalism and Its Application to the Study of Effects of Laser Pulses in Multiphoton Processes. Chemical Physics Letters, 225, 46-54. https://doi.org/10.1016/0009-2614(94)00607-5

[11] Breuer, H.P., Dietz, K. and Holthaus, M. (1991) Selective Excitation of Molecular Vibrations by Interference of Floquet States. Journal of Physics B: Atomic, Molecular and Optical Physics, 24, 1343-1357. https://doi.org/10.1088/0953-4075/24/6/021

[12] Lemos, N.A. and Natividade, C.P. (1987) Harmonic Oscillator in Expanding Universes. Nuovo Cimento, 99, 211-225. https://doi.org/10.1007/BF02726583

[13] Paul, W. (1990) Electromagnetic Traps for Charged and Neutral Particles. Reviews of Modern Physics, 62, 531-540. https://doi.org/10.1103/RevModPhys.62.531

[14] Brown, L.S. (1991) Quantum Motion in a Paul Trap. Physical Review Letters, 66, 527-529. https://doi.org/10.1103/PhysRevLett.66.527 
[15] Hashemloo, A., Dion, C.M. and Rahali, G. (2015) Wave-Packet Dynamics of an Atomic Ion in a Paul Trap: Approximations and Stability. arXiv:physics1312.2419v6, 1-20.

[16] Breuer, H.P. and Holthaus, M. (1989) Adiabatic Processes in the Ionization of Highly Excited Hydrogen Atoms. Zeitschrift für Physik, 11, 1-14. https://doi.org/10.1007/BF01436579

[17] Janati Idrissi, M., Fedoul, A., Achkar, Y., Chatwiti, A. and Sayouri, S. (2011) Squeezing in Floquet States and Quasi-Energies of Harmonic Oscillator Driven by a Strong Periodic Field. African Journal of Mathematical Physics, 10, 21-30.

[18] Janati Idrissi, M., Fedoul, A., Sayouri, S. and Amila, I. (2020) Anharmonic Potentials Analysis through the Floquet Representation. Journal of Applied Mathematics and Physics, 8, 184-195. https://doi.org/10.4236/jamp.2020.81014

[19] Lochak, G. and Thioun, M. (1969) Sur une méthode générale de perturbation en mécanique ondulatoire et son utilisation dans les problèmes de resonance. Journal of Physics, 30, 482-496. https://doi.org/10.1051/jphys:01969003005-6048200

[20] Rabi, I.I., Ramsey, N.F. and Schwinger, J. (1954) Use of Rotating Coordinates in Magnetic Resonance Problems. Reviews of Modern Physics, 26, 167-171. https://doi.org/10.1103/RevModPhys.26.167

[21] Jàuregui, R. (2001) Nonperturbative and Perturbative Treatments of Parametric Heating in Atom Traps. arXiv:physics/0104075v1, 1-13. https://doi.org/10.1103/PhysRevA.64.053408

[22] Zhang, W.M., Feng, D.H. and Gilmore, R. (1990) Coherent States: Theory and Some Applications. Reviews of Modern Physics, 62, 867-924. https://doi.org/10.1103/RevModPhys.62.867

[23] Hai, W., Huang, S. and Gao, K. (2003) Wave-Packet Trains of a Time-Dependent Harmonic Oscillator. arXiv: quant-ph/0301156v1, 1-4.

[24] Um, C.C., Choi, J.R., Yeon, K.H. and George, T. (2002) Exact Quantum Theory of the Harmonic Oscillator with the Classical Solution in the Form of Mathieu Functions. Journal of the Korean Physical Society, 40, 969-973. 


\section{Appendices}

$\hbar$ : Reduced Planck's constant.

$\left|\varphi_{n}(t)\right\rangle$ : Eigenstates of the operator $R$.

$E_{n}$ : Eigenvalues of $R$ (Quasi-energies).

$|n\rangle:$ Sates of the unperturbed system.

$\left|\psi_{n}(t)\right\rangle$ : Floquet states.

$p$. Impulsion operator.

$q$ : Position operator.

$m$ : Mass of the particle.

$\delta:$ Parameter of the trap chamber.

$\omega_{0}$ : Unperturbed oscillator frequency.

a: Annihilation operator.

$a^{+}$: Creation operator.

$\operatorname{Im} \eta_{1}(t)$ : Imaginary part of $\eta_{1}(t)$.

$\operatorname{Re} \eta_{2}(t)$ : Real part of $\eta_{2}(t)$.

$\left|\eta_{1}\right|$ : Modulus of $\eta_{1}(t)$.

$\eta_{2}^{\star}(t)$ : Complex conjugate of $\eta_{2}(t)$. 